\title{
Cognitive dialogue as a factor of enhancing the quality of education in the conditions of international cooperation in the sphere of higher education
}

\author{
Larisa Mukhametzyanova ${ }^{1}$ \\ Institute of Pedagogy, Psychology and Social Problems, Isaev Str., 12, 420039 Kazan, Russia
}

\begin{abstract}
The relevance of the article is caused due to the need to define the role of a cognitive paradigm for enhancing the quality of higher education in the conditions of international cooperation contributing to the development of the subjects' of educational process possessing the humanforming, semantic priorities. The purpose of article is to reveal the humanforming individual reserves and mechanisms of cognitive dialogue between the teacher and the students on the basis of social-cognitive approach as the theoretical-methodological strategy determining and developing humanistic semantic formations and personality's value transformations. The article reveals the principles of social-cognitive approach: human-conformity and harmonious proportionality, and the rules for their realization; cognitive instruments of democratization and humanization of education are described on the basis of cognitive dialogue between the teacher and the students as an informative exchange of rational and emotional information. The author defines the importance of emotional intelligence as a link between development of humanistic meanings, value understanding, understanding and regulation of the subjects' of education cognition and emotions in cognitive dialogue affecting success of their socio-cultural interaction. The developmental stages of dialogical selfknowledge in the course of cognitive dialogue are defined in the integral unity of logical and emotional, and value coordinates based on the material of art works. The article is intended for University-teachers and researchers in the field of international cooperation in the sphere of education.
\end{abstract}

\section{Introduction}

The relevance of the research is caused due to the fact that the special position in the development of personality's new values, and cultural and behavioural practices in the era of digitalization is taken by value and semantic priorities of an educational system which defines that the learner is not only the holder of the professional knowledge, skills, but also the person possessing special human qualities and properties which allow him (her) to

*Corresponding author: $\underline{\text { lar_ur@list.ru }}$ 
participate in the socio-cultural interaction. The outstanding German teacher-humanist of the middle 19 - $^{\text {th }}$ century Friedrich Adolf Wilhelm von Disterveg defines the education purpose as follows: "In each individual, in each nation humanity-oriented worldview has to be brought up: this aspiration leads to the noble universal purposes" [1, P. 136].

The cognitive paradigm of education is aimed at establishment of the value and semantic priorities of an educational system of higher education institution providing realization of its humanistic potential in the process of development the Human of knowledge in the era of digitalization. "We believe that it is possible to talk about the appearance of the cognitive paradigm of education which is based on the appeal to ways, types and technologies of processing information by the human for the purpose of creation of a personal system of representations, knowledge and competences for the definite subject sphere and one's own system of worldviews with transfer to generation of knowledge. The ability to transfer information into knowledge, "to create" knowledge, to generate new technologies is the main characteristic of the human capital requested by the economy. Subsequently, there appears a question to the educational system of higher education institution: How is it possible not to lose the personality and the Human in the era of digitalization? [2, P. 8]. The social-cognitive approach treated by us in terms of transformation of the processes for professional socialization and inter-personal interaction in the context of personality's value and semantic formations development acts as the intrinsic engine of a cognitive educational paradigm. "Social-cognitive approach is a theoretical and methodological strategy determining and developing personality's semantic formations and value transformations for the purpose of creation of his (her) humanforming and social qualities in the course of socio-cultural interaction" [3, P. 31].

In the era of digitalization, it is essentially important to create a significantly new organization of pedagogical processes and conditions for the development of the personal cognitive mechanisms providing formation of universal students' qualities (self-skills), the range of which will promote the finding of the solution for professional tasks. Namely "Human-forming" type of education is the global innovative reserve capable to change a situation in the modern educational organization in the direction of its humanization cardinally.

One of the key positions of social-cognitive approach is as follows: the personality comprehends the meanings in the reality of the objective world, culture and the speech by means of the social interaction defining an initial context of teaching in the educational organization of the higher education as determination of personlity's value and semantic transformations in the course of formation his (her) human-forming qualities and professional characteristics. The study and the human's development become essentially important in the context of realization of social-cognitive approach in variety of its cultural and semantic relationships with rapidly-transformed "digital" reality.

Key concepts of social-cognitive approach:

- social-cognitive approach - the theoretical and methodological strategy defining cultural and semantic, and value and humanistic priorities of the personality in the context of their harmonious proportionality with the humanistic settings of the society;

- the principle of human-conformity defines the existing regulatory of educational process as its cultural and semantic kernel of an individual conceptual sphere that assumes an accent on semantic formations and value transformations of the personality;

- the principle of harmonious proportionality opening a humanistic matrix for the development of the harmonious personality in the integral unity of cognitive stability, activity, clarity, "symmetry"; proportionality of personality's value and semantic settings to socio-cultural values; 
- humanistic cognition as the manifestation of human's intellectual abilities including humanistic personal and value understanding in the course of socio-cultural interaction of the higher education actors;

-concept is treated as a universal, a meaning, an idea which is represented in the culture, set and verbalized, forming a system of personal and value, and semantic integrity with implementation of cognitive and emotional components of cognition;

- individual conceptual sphere represents an integral unity of cultural universals, personal semantic settings, the human's emotional value connotations comprising the essence of his (her) personal and professional picture - a worldview.

Basing on the conceptual ideas of social-cognitive approach, we tried to reveal an intrinsic form of the subject - subject interaction in the educational process of higher education institution in the framework of a cognitive paradigm, suggesting a humanforming reflection of information and knowledge. Dialogue is considered to be a productive type of the voluntary, equal inter-subject inter-relations according to which the interacting partners are able to reveal new information in the course of communicative acts and are constantly changing, at the same time having kept their sovereignty and identity $[4,5,6]$

\section{Materials and methods}

The methodological settings of our research are social-cognitive approach and the principles of its realization: the principle of a human-conformity and the principle of harmonious proportionality.

Purposeful approach settings: it is aimed at mastering the person's culture-conformity, nature-conformity and professional meanings within higher education, their transformation to one's own concepts on the basis of an individual ability to process new information and generate knowledge, and develop an individual conceptual sphere. An effective development of this ability at a human depends on his (her) cognitive features mechanisms of cognition, which define the acquiring of scientific knowledge in the cultural and value dialogue of times, the conceptual points of view defining an image of an individual and professional worldview.

Application of social-cognitive approach in education provides involvement subjects' of education cognition in the humanistic context including humanistic, personal and value understanding of one's own image and the professional activity with a support on initial, social settings. Any professional sphere continuously recreates a number of the professional situations constructed on the basis of human's cognitive resources which determine the level of professionalism of students' activities and their social responsibility for implementation of professional activities. It assumes the formation of a certain "ideal" (an action pattern, an image) necessary for modern society which is transformed taking into account individual conceptual sphere - the opportunities for personal, intellectual and physical development of the specific human.

Advantages of social-cognitive approach in practice of the higher education are defined by the possibilities of reflecting the picture of an outside world, social norms and the practices of social interaction, the subject professional sphere on students' cognitive individual development and formation of his(her) individual conceptual sphere. Implementation of human-forming, individual reserves and mechanisms of selforganization in educational activity contributes to the development of the personal and professional concepts and values, defining the orientation of semantic settings, basic and instrumental norms of activity in the context of cultural representation. The perspectives of application of social-cognitive approach can be traced in a possibility of studying impact of social interaction not only on cognitive processes, but also on the personality's semantic formations determining his (her) social behavior and professional positioning. 
Cognitive dialogue is based on the basis of the following principles:

- the principle of a human-conformity causing the focus on the human (his opportunities, his abilities, his needs and his life mission) on design and realization of higher education [7];

- the principle of harmonious proportionality revealing a humanistic, developmental matrix of the harmonious personality in the integral unity of cognitive stability, activity, clarity, "symmetry"; proportionality of personality's value and semantic settings to sociocultural values.

The pedagogical principle is realized in rules. Rules act as additional recommendations to a definite principle.

The principle of human-conformity in the course of cognitive personal development assumes implementation of the following rules:

- identification in the content of education the developmental problems of human's spiritual essence;

- accounting of the cultural and humanistic potential of the educational environment influencing cultural and semantic coordinates of the student's identity;

- determination features of the students' original world taking into account his emotional and sensual sphere, informative abilities, personal interests;

- humanity-oriented focus of the relations within an intelligent community of educational process subjects.

These rules of realization of the human-conformity principle are based on the humanistic reflection, allowing subjects of educational process to develop their personal qualities, including conceptual humanistic understanding and semantic assessment of themselves and the surrounding world.

The following principle - the principle of harmonious proportionality revealing a humanistic, developmental matrix o of the harmonious personality in the integral unity of cognitive stability, activity, clarity, "cultural symmetry"; proportionality of value and semantic settings of the personality to socio-cultural values.

The principle of harmonious proportionality pre-supposes implementation of the following rules:

- determination in the content of education the problems of formation planetary consciousness and elements of all-human culture;

- harmony of a society-orientation (external plan of cognitive development) and selforientation (internal plan of cognitive development) as two into-coherent and interconnected modes of personality's orientation on oneself and on the society as two vectors of humanistic self-creation;

- coherence of two key manifestations of harmony - states of parts and their compensatory effect for the purpose of identification of symmetric coordination between personal and social meanings of human's cognitive development;

- correlation of the idea of rationally-arranged world to the ability to get an emotional experience displaying an essence of a proportional ratio of human's feelings and reason.

For the purpose of verification the founded solutions of the objectives, the following methods of the research were used: the problem-based analysis and synthesis of the ideas, allowing to comprehend the category "cognitive dialogue", analogy, generalization, specification, abstraction, idealization, extrapolation of the revealed trends, regularities and developmental mechanisms of cognitive dialogue between the teacher and the students in higher education institution on the possibility of their implementation in the practice of educational institutions. 


\section{Results}

We treat the cognitive dialogue in the framework of basic positions of social-cognitive approach as a factor of international cooperation development. The principles of its realization are: the principle of human-conformity and the principle of harmonious proportionality. This dialogue assumes identification of the student's attitude towards knowledge, stimulates the establishment of a personal position, creates the atmosphere of mutual understanding, trust, openness, provides a form of self-creation of as the learning person on the basis of interrelation between informative, value and semantic, and conceptual aspects of self-consciousness $[8,9]$.

Dialogue is a principally different phenomenon comparing to an exchange of monologues as a form of extended statements marking activity of the subjects of education. The monologues can be both appealed, and distinguishable. The appealed monologues influence the addressee, but they do not imply a speech response. The appealed monologues are treated as a center of personal meanings, steady and deep. They comprise the integral layer of culture.

Distinguishable monologues are the statements which are carried out by a person who is alone (literal) or in psychological isolation. These are diary writings, providing "speaking" for themselves.

Distinguishable monologues are an integral part of human life. According to the scientific belief of V. Hayrapetyan, "to think - means, first of all, to speak with oneself" [10]. These monologues are integrally connected with what Yu. M. Lotman called "autocommunication" which is the cornerstone of the situation "I-I", but not "I-HE". "European culture, - the scientist claimed, - consciously and purposefully is guided by the "I-HE" system, but there are cultures focused mainly on auto-communication (countries of the East): they are capable to show a great spiritual activity, however they often turn to be less dynamic" [11, P. 89]. Any participant of the dialogue, using semantic auto-communication after each statement, changes his (her) own state due to the information gain in the course of an infinite meaning-formation.

A compulsory condition for the dialogue - a question-answer form of interaction. However, questions and answers can be multi-purposed and multi-focused. Questions of information character are programming a definite answer which almost does not change neither asking, nor answering. M. Bakhtin and M.S. Kagan [12,13] consider the defining sign of dialogue to be an equal interaction of partners in the course of which there occurs a mutual exchange and the birth of new general sense. Participants of an equal dialogue after a statement change their own state.

Special value in a perspective of consideration of a dialogue as a type of equal subject inter-relations, promoting maintaining their sovereignty and identity, and a monologue as an integral reasonable side of human life "to think - means, first of all, to speak with oneself" is given to a cognitive dialogue between the teacher and students.

M.M. Bakhtin wrote that "the truth is not born in the head of a certain person, it is born between the people who are searching together in the course of their dialogical communication" [12, P. 16]. Cognitive dialogue assumes identification of the students' attitude towards knowledge, promotes manifestation of a personal position, creates the atmosphere of mutual understanding, trust, openness, provides a form of creation oneself as the learning person on the basis of inter-relation of informative, value and semantic, and conceptual aspects of consciousness. Dialogue is based on the statements of different people and is carried out by a chain of the laconic semantic statements called remarks. Socrates said: "If you want to talk to me, use shortage of words" [14]. The ability to conduct a dialogue is a special sphere of speech culture where keenness to the interlocutor, flexibility of a thought, compliance between ability to speak (responding to a moment 
situation) and ability to listen attentively to words from the surrounding person. Personal dialogue communication is realized with the teacher who, as a rule, is treated by the students of higher education institutions as a central person, and is the master transferring professional secrets in the course of individual interaction so called "from hand to hand".

Cognitive dialogue between the subjects of education is realized in the humanistic context including humanistic, personal and value understanding of one's own image and the professional activity with a support on initial social settings in practice of the higher education. It defines freedom of personal expression, an opportunity to support one's own rights, sincerely and free show the emotional states, but, at the same time, to preserve one's own self-esteem and dignity, and respect for other personality. These human-forming manifestations define the status of an intelligent person: "An intelligent person is a person possessing intellectual decency. The basic principle of intelligence is an intellectual freedom, freedom as moral category. An intelligent person is not free only from the conscience and from his (her) thought ". [15, P. 7].

In modern conditions of rapidly-happening changes essentially important is the ability to react quickly on changes, to adopt to new conditions. Intellectual skills, the ability to understand correctly people and their acts, to assess the situation the person gets into, the establishment and strengthening the inter-relations with the surrounding people are considered also relevant. The cognition processes including imagination, perception, memory, way of thinking in cognitive dialogue are based, first of all, on creation of an emotional image which becomes a specific instrument of cognition of personality's both external, and inner world. In the complicated era of digitalization, the most effective way of creation the worldview for subjects of educational process is implementation of interaction between traditional models of emotionally-figurative perception with the new opportunities connected with modern means of obtaining information. Enlargement of an information field thanks to availability and speed of receiving audio and video, allows us to address the reference records of master-classes that become some kind of "school for developing professional skills" and open the new horizons of cognition. The emotional conditionality of cognitive dialogue determines the need for definition the instruments providing value understanding and semantic regulation on the basis of integration subjects of education' cognition and emotions [16].

The emotional intelligence serves as an instrument for development of humanistic meanings, value understanding, understanding and regulation of cognition and emotions of the subjects of education affecting success of their cognitive dialogue. Emotional intelligence is a complete dynamic formation integrating cognitive and emotional, and figurative components of cognition; providing optimistic value understanding, understanding and semantic regulation of one's own emotions and emotions of surrounding people and affecting success in a cognitive development of individual conceptual sphere $[15,16,17]$

The development of emotional intelligence can happen simultaneously to the development of cognitive abilities at all stages of teaching, at the same extent, contributing to the human's comprehensive development in the course of teaching, and further affects success in its personal and professional activity.

In the respect of educational reserves of emotional intelligence, it acts as follows: accounting of students' emotional condition when choosing pedagogical influence, prevention of a didaktogenii and emotional burning out, preservation and restoration of the lost trust relations in the process of inter-subject interaction. Respectively, there is a need for enlightenment concerning a role of emotional intelligence as a component of individual results of students' teaching. Besides, there appears a question of implementation emotional intelligence as a resource of individual development by the teachers. 
Thus, the emotional intelligence mediates a humanistic-centered focus of interaction between the subjects of educational environment, promotes the realization of an individual approach in the process of individual development and in the course of teaching and upbringing, raises the level of teacher's personal and professional maturity. Accentuation on students' attention, his emotional and value perception of knowledge leads to its strong assimilation. It means that the human is not able to acquire something and study it comprehensively until he (she) does not comprehend information completely and emotionally over-estimate it. Development of emotional intelligence can happen simultaneously to the development of cognitive abilities at all stages of teaching, to the same extent contributing to the human's comprehensive development in the course of teaching, and further affecting success in its personal and professional activity.

Realization of self-development, self-cognition in the course of cognitive dialogue is connected with continuous, creative search of the new decisions revealing space for imagination, and allowing to create an individual product, not to be afraid of courageous, unexpected turns in the course of finding solution for professional tasks. Therefore, there should be an attraction of such a creative "engine" which would contribute to the development of dialogical self-cognition in the conditions of cognitive dialogue between the teacher and the students. The art providing an emotional and image component of cognitive dialogue acts as this powerful engine. It is possible to define the following, developmental stages of dialogical self-cognition in the course of cognitive dialogue in its integral unity of logical and emotional, and value coordinates based on the material of art works.

The necessary condition for the first stage - identification, and recognition of "a stranger" in "oneself" and also an opportunity to see "oneself" in "a stranger". The need for personal identification is especially essential when appears during the crisis periods. In psychology these crises are connected with the definite age periods or with social cataclysms. Value and informative mechanisms of individual perception are caused by these two features: subjective and age features, and objective and social features. Students search for the support in getting answers to the appearing questions most often in the "mirrors" (reflections) in people's estimations, and patterns. Therefore, the author's personality and heroes of the art work can become this "mirror". O. Wilde defined that "the art is the mirror reflecting the one who looks in it" [20, P. 9]. Leaving apart with an expansion of self-thinking "I", it opens almost an unlimited space for an individual selfdevelopment. Having left the real mirror its primary function - to set our appearance, art serves as a "mirror" of an incomparably greater optical power which suggests a real assistance to the personality to see and understand in itself the essence, looking in heroes of the art work as in the reflection. At the same time, a distinctive sign of this process is that it is aimed at bringing personality's spiritual world to a state of semantic harmony.

At the first stage the recognition of positions, the heroes' patterned points of view are significant. The problem of an internal dispute as self-cognition became one of the main in works of the philosopher and literary critic M. Bakhtin, first of all, appearing in his work "A problem of creativity in art works of F.M. Dostoyevsky". M. Bakhtin shows that a situation of "non-recognizing - recognizing" themselves is a situation of the transition of an "external" dialogue with the surrounding people, and "internal" dialogue with himself. Nastasia Filippovna "recognizes" herself in Rogozhin and in Myshkin, Ivan Karamazov - in Alyosha and Smerdyakov, Raskolnikov - in Sonya and Svidrigaylov. However, "to recognize oneself" in another person means simultaneous recognition of own discrepancy, and the worlds which sometimes are mutually excluding each other [21].

Admission of these worlds as "belonging to oneself" at the same time has to mean leading them under some general notion. Therefore, the next stage of development for dialogical self-cognition - from simple recognition features in another person to admission 
of the general basis of dialogue, despite external distinctions; it is the period of a depersonification of disputing parties. At this stage it is possible to speak about communication of one personality with the other, but not about communication between representatives of various age and social groups.

There exists the other form of the general which can stand for an original basis of an internal dialogue. This is the definite and general, assuming not only the presence of the general signs, but the existence of the general law connecting various individuals within social wholeness, identification of personalities' integral unity, but not the uniformity.

The personality, realizing oneself the holder of definite and general, does not treat oneself as somebody "finished", and "completely realized". The person creates the world, by himself (herself). "To be a contemporary person means to create the time, but not to anticipate it. Yes, it is necessary to reflect it, but not as a mirror, do it as a shield. To be a contemporary person means to create the time", - M. Tsvetaeva wrote [22].

The highest stage is characterized by such form of dialogue according to which the communication between the arguing parties is based on a definite and general dialogue which can become not just an "understanding" of oneself, but a form of self-creation. At this stage of dialogical model, the informative, value and design aspects of consciousness coincide. Dialogue becomes the personality's way of life.

Creative processing, reconsideration of a huge number of information obtained by modern students is carried out by means of their inclusion in real process of practical activities thanks to which a cognitive dialogue is performed. The role of the teacher in higher education institution in the process of organization of cognitive dialogue is significant, as well. The ability of the teacher to present information from the innovative, extra-ordinary point of view, the ability to focus students' attention on the problem field of inexhaustible knowledge of the world gives the grounds for creative dialogue which is developed on the basis of human's statements and is carried out by a chain of laconic statements. The ability to conduct dialogue is a special sphere of speech culture where it is "required" from the personality keenness to the interlocutor, flexibility of a thought, compliance between ability to speak (responding to a moment situation), and the ability to listen attentively to the words of surrounding person. A personal dialogue communication is realized with the teacher who serves, as a rule, the central person for students of higher education institutions and the master transferring professional secrets in the course of individual interaction, so called "from hand to hand".

In the course of cognitive dialogue in the framework of a cognitive paradigm the teacher's position significantly changes: the role of the teacher of the higher school is gradually transformed to a role of the consultant, stalker, in the literary sense of this word. The focus is shifted to students' self-control, self-management. Cognitive dialogue provides not just an "understanding" of oneself, but a form of self-creation as the learner on the basis of inter-relation of informative, value and semantic, and conceptual aspects of consciousness. Such dialogue becomes the personality's way of life, therefore the teacher (who is the holder of cognitive instruments) is the teacher capable to fruitful informative search, fast adoption of original decisions in unusual situations feeling emotional comfort from success of knowledge implementation and from an opportunity to generate new knowledge, using mastered ways and methods of operating, and the internal personal resources. The teacher, who, provides formation students' cognitive and thinking, and humanistic-focused, behavioural strategies forming the basis of the identity for Human of knowledge - the thinking person possessing human-conformity ("intellectual decency") and harmonious proportionality ("intellectual freedom as a moral category").

The cognitive dialogue taking into consideration specific features of dialogical educational space of higher education institution is a condition for the development of 
teachers' ability to reveal oneself in the speech by means of dialogical and monological, emotional and semantic figurativeness.

Teacher's emotional and cognitive self-realization is connected with the hermeneutical direction according to which, teachers should prepare students for perception, adjust them on experience and empathy. The essential position of hermeneutics, intrinsic for the educational environment, is the approval of dialogical character of understanding. The main thing in the "understanding" education is an opportunity and desire to reveal to something that cannot be told, and an ability to experience this openness.

An important link in teacher's "understanding education" in the framework of cognitive dialogue is the emotional identification, the phenomenon functioning at various stages of educational process acts. At the first stage - appearance of a pedagogical idea - emotional identification of the teacher acts in the most general view. Here the principles of influence are considered. At the second stage - development of the idea - emotional identification acts more actively, as a result, various possible options in the system of pedagogical communication tend to appear. At the third stage - the embodiment and implementation of the idea - emotional identification acts as the general principle of feedback in communication. At the fourth stage of pedagogical creativity the emotional identification suggests a real assistance to the teacher to present results of the activity. Here emotional identification acts as an estimating and specifying element. The efficiency of emotional identification is checked by laws of pedagogical logics and acts as the kind of his figurative pedagogical thinking with an accent on emotional-empathy component of teacher's selfrealization, developing on the basis of enlargement of an information field thanks to availability and speed of receiving audio and video, which allows us to address reference records of master classes that become some kind of "school for the development of teacher's professional skills" and opens the new horizons of cognition.

\section{Conclusions}

Cognitive dialogue between the teacher and students is defined as a factor of enhancing the quality of higher education in the conditions of international cooperation in the sphere of higher education. On the basis of providing a humanistic context of the subject - the subject interaction, it defines humanistic, personal and value understanding of one's own image on the basis of inter-relation between informative, value and semantic, and conceptual aspects

of consciousness with a support on initial social settings in practice of higher education.

In the article, the human-forming individual reserves and mechanisms of cognitive dialogue between a teacher and the students on the basis of social-cognitive approach as the theoretical-methodological strategy, determining and developing personality's humanistic semantic formations and value transformations. The principles of its realization are revealed: the principle of human-conformity and the principle of harmonious proportionality.

In the conditions of a cognitive dialogue, the subjects of higher educational environment are capable to fruitful informative search, feeling emotional comfort from the success of knowledge implementation, and realizing cognitive and thinking, and humanisticallyfocused behavioural strategies of life activities in the framework of a cognitive paradigm.

\section{References:}

1. A. Disterveg Selected pedagogical works (1956)

2. E. Levina, L.Ju. Mukhametzyanova Kazan Ped. J., 3(40), 8 (2020) 
3. R. Gilmeeva, A. Kamaleeva, A. Kats, E. Levina, V. Maslennikova, L.Mukhametzyanova, T. Tregubova, L. Shibankova Cognitive pedagogy (2020)

4. N.D. Feshbach, Parental empathy and child adjustment/maladjustment. (1987)

5. C.R. Rogers A theory of therapy, personality and interpersonal relationships as developed in the client-centered framework. (1959)

6. M. Davis Empathy: a social psychological approach (1966)

7. A. Khutorskoy Mess. of Volgograd state ped. univ., 6, 4 (2009)

8. S. Belova Nat. Ed., 7, 103 (2001)

9. S. Yachin Human, 5, 142 (2001)

10. V. Airapetyan Hermeneutic approaches to Russian word (1992)

11. Ju. Lotman Semiosphera (2000)

12. M. Bakhtin Esthetics of verbal art (1986)

13. M. Kagan Philosophy of culture (2019)

14. Platon Dialogues (2017)

15. D. Likhachev New world, 2, 3 (1993)

16. A.Hargreaves Teaching and Teacher Ed, 14 (8), 835 (1998)

17. S. Titsworth, M.M.Quinlan, J.P. Mazer Comm. Ed., 59, 431 (2010)

18. A. Bechara, D. Tranel, A.R. Damasio The Handbook of Emotional Intelligence. Poor judgment in spite of high intellect: Neurological evidence for emotional intelligence. (2000)

19. P. Salovey, D. Grewal Current Dir. in Psych. S., 14, 281 (2005)

20. O. Wilde Portrait of Dorian Grey (2017)

21. M. Bakhtin Problems of poetics by Dostoevsky (2002)

22. M. Tsvetaeva Poet and epoch (2012) 